\title{
A New Definition of Short-circuit Ratio for Multi-converter HVDC Systems
}

\author{
Dengfeng Liu ${ }^{\dagger}$, Dongyuan Shi* and Yinhong Li*
}

\begin{abstract}
In this paper, a new definition of short-circuit ratio concept for multi-converter HVDC systems is proposed. Analysis results of voltage interaction between converters show that the reactive power-voltage characteristic of a converter has a dominant effect on voltage interaction level compared with its active power-voltage characteristic. Such a relation between converter reactive power and voltage interaction level supports taking the former into account in the definition of short-circuit ratio concept for multi-converter systems. The proposed definition is verified by the method of maximum power curve for various system configurations. Furthermore, a formula to calculate transient overvoltage for multi-converter systems is derived based on the proposed definition, and the efficiency of the derived formula is verified.
\end{abstract}

Keywords: High voltage direct current, Multi-converter system, Short-circuit ratio, Multi infeed interaction factor, Converter reactive power-voltage characteristic, Transient overvoltage

\section{Introduction}

Since the year of 1954, high voltage direct current (HVDC) systems have offered new dimensions for long distance overhead bulk power transmittal, interconnection of different frequency systems or asynchronous systems, and undersea cable systems. In recent years, the maturing of line commutated technology, the liberalization of electrical market, and the rapidly growing of electrification in developing countries such as China have contributed to an increasing consideration of HVDC applications [1]. As a result, system configurations wherein multiple HVDC converters are connected into a common ac network, termed as multi-converter HVDC systems [2], become common. In most grids, they appear as the configuration wherein all the converters operate as inverters, termed as multi-infeed HVDC systems [2, 3]. However, another configuration in which one converter operates as an inverter while a neighboring converter as a rectifier, termed as rectifier-inverter HVDC systems [2], appears gradually at an ac system that covers a vast geographic area with a large amount of variable generation [4]. In Chinese Center Grid, an inverter station was planned to consume the wind energy from Xinjiang Province [5], which came true in 2014 and shaped a rectifier-inverter HVDC system with several existing rectifiers.

There are some technical aspects related to multiconverter HVDC systems that are caused by the interaction

$\dagger$ Corresponding Author: State Key Laboratory of Advanced Electromagnetic Engineering and Technology, Huazhong University of Science and Technology, Wuhan, China. (hustliudfeng@163.com)

* State Key Laboratory of Advanced Electro-magnetic Engineering and Technology, Huazhong University of Science and Technology, Wuhan, China. (dongyuanshi@163.com)

Received: August 4, 2014; Accepted: June 18, 2015 between converters and can ultimately influence the overall performance of the integrated ac/dc systems. An important indicator for HVDC basic performance analysis has been the short-circuit ratio (SCR) and effective SCR (ESCR) concept. Although not indicative of all effects including power voltage instability, transient overvoltage (TOV), commutation failure and fault recovery, and harmonic interaction, its simplicity has continued to make it useful for the level of performance expected from an HVDC system [6-10].

For multi-infeed HVDC systems, some factors describing the interaction between inverters, such as multi infeed interaction factor (MIIF), are proposed, and then the SCR and ESCR concept applicable to single-infeed HVDC systems is extended to multi-infeed systems based on these interaction factors $[3,11]$. Parametric study results by the method of maximum power curve (MPC) show that critical values of multi-infeed interactive ESCR (MIESCR) are about 1.5 under various system conditions, which is in accord with the critical value of ESCR in a single-infeed system [12].

For rectifier-inverter HVDC systems, critical values of MIESCR are found varying greatly around 1.5 , which indicates that the definition of MIESCR is not valid any more. Then, a definition of SCR concept for rectifierinverter systems is proposed based on the method of parameter fitting [13]. However, the definition in [13] is found by the paper to be invalid in situations where inverters are very close to rectifiers in electrical distance. Moreover, it may encounter potential inconvenience caused by revaluing the exponential parameter in new situations, since the value is obtained by parameter fitting and hence related to system parameters.

In this paper, a definition of SCR for multi-converter 
HVDC system is presented based on discussions about converter equivalence and on analysis of voltage interaction between converters. It found that the reactive powervoltage characteristic of a converter has an absolutely dominant effect on voltage interaction level compared with its active power-voltage characteristic. When a voltage interaction indicator, such as MIIF, is used as the weighting factor in the process of converter equivalence, the relation between the reactive power of the equivalent converter and MIIF should be taken into account in the definition of SCR concept for multi-converter systems.

The outline of this paper is organized as follows. In Section 2, current definitions of SCR for various HVDC system configurations are discussed, and the procedure of converter equivalence is analyzed. Section 3 analyses mathematically characteristics of voltage interaction between converters based on the analytical definition of MIIF. In Section 4, after discussions about the significance of converter reactive at both static and dynamic states for the whole system's performance, a definition named QMCESCR for multi-converter HVDC systems is proposed. Then, the proposed definition is validated with various HVDC system scenarios based on MPC in Section 5. In Section 6, a formula to calculate TOV for multi-converter systems is derived based on QMCESCR, and the efficiency of the formula derived is verified.

\section{Current Definitions of SCR for HVDC Systems}

\subsection{Single-infeed HVDC systems}

A single-infeed HVDC system employs ESCR to assess whether or not the ac system is sufficiently strong to support the operation of the HVDC system connected. The definition of ESCR for single-infeed systems represented by the model in Fig. 1 is addressed mathematically as (1).

$$
E S C R=\frac{S_{S C C}-Q_{c}}{P_{d c N}}
$$

In (1), $S_{S C C}$ represents the short circuit MVA of the ac system at the converter ac bus, $P_{d c N}$ is the MW rating of the converter, and $Q_{c}$ is the shunt compensation at the rated voltage magnitude.

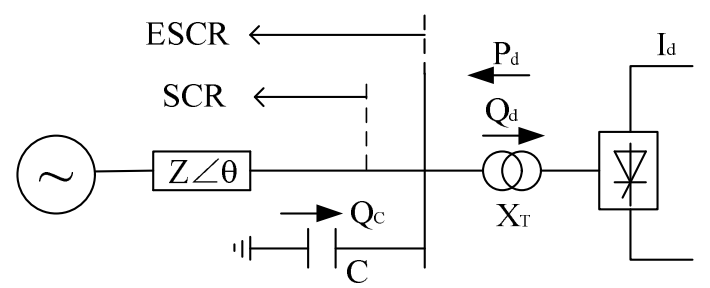

Fig. 1. The schematic diagram of a single-infeed HVDC system
The critical ESCR (CESCR) describes a system in which the maximum available power (MAP) of a de link occurs at dc current equal to 1.0 p.u.. The method of MPC shows that the value of CESCR is about 1.5 for an inverter with constant extinction angle control at about 15 to 18 degrees in a single-infeed link modelled by the CIGRE HVDC benchmark model [14].

\subsection{Multi-infeed HVDC systems}

In the multi-infeed context, due to the common sharing of all the HVDC links connected to the same ac system on the system strength, the calculation of ESCR should take the influence of power injections from other links into account. There have been two definitions of ESCR proposed for multi-infeed HVDC systems, which consider the influence of adjacent links by weighting their active power injections with corresponding factors. One definition, named as MESCR and showed as (2), is based on the ratio of elements of the node impedance matrix retaining inverter converter ac buses [11], and the other, named as MIESCR and showed as (3), is based on MIIF [3]. In (2), $\tilde{Z}_{e . i j}$ is the i-th row and j-th column element in the node impedance matrix in p.u., and $\tilde{P}_{d c N . j}$ is the rated power of the $\mathrm{j}$-th converter in p.u..

$$
\begin{aligned}
\operatorname{MESCR}_{i}= & \frac{1}{\sum_{j=1}^{n} \tilde{\mathrm{Z}}_{e . i j} \cdot \tilde{P}_{d c N . j}} \\
\operatorname{MIESCR}_{i}= & \frac{S_{S C C . i}-Q_{c . i}}{\sum_{j=1}^{n} \operatorname{MIIF}_{j, i} \cdot P_{d c N . j}}
\end{aligned}
$$

Investigation results show that the critical values of MIESCR are essentially the same as the value of CESCR for single-infeed systems [12]. The consistent phenomena of critical values between ESCR and MIESCR indicate that the ac system strength seen at the converter under consideration in a multi-infeed system is equivalent to that in a single-infeed system. Consequently, the converter and the corresponding HVDC link may have the same characteristics as that at single-infeed systems, such as power-voltage stability and temporary overvoltage. Moreover, the consistency verifies the effectiveness of MIIF as well as MIESCR in assessing ac system strength for multi-infeed HVDC systems.

\subsection{Rectifier-inverter HVDC systems}

One natural attempt is to extend the definition of MIESCR to rectifier-inverter HVDC systems. Investigation results show that the critical values of MIESCR for the rectifier-inverter system under different system conditions vary greatly around 1.5 [13], which are not consistent 
with those in multi-infeed HVDC systems and will cause confusion at the estimation of ac system strength by MIESCR value.

An improved definition of MIESCR for rectifier-inverter HVDC systems, based on the methods of MPC and parameter fitting, is proposed and shown as (4) [13]. The values of $\alpha$ and $k$ depend on the operation type of a converter. When the converter operates as a rectifier, $\alpha$ values 2.2 and $k$ values 1 . While as an inverter, $\alpha$ values 1 and $k$ values 0 , which makes (4) become the same as (3).

$$
\operatorname{MIESCR}_{i}=\frac{S_{S C C . i}-Q_{c . i}}{P_{d c N . i}+\sum_{j=1, j \neq i}^{n}(-1)^{k} M I I F_{j, i}^{\alpha} \cdot\left|P_{d c N . j}\right|}
$$

If considering a special scenario in which a rectifier and an inverter with the same rated power have a very close electrical distance (it means that the value of MIIF approaches to 1), the value of MIESCR calculated by (4) will be very large, since the denominator in the left side of (4) values nearly to 0 . Then, we can conclude by the enormous MIESCR value that the ac system will be very strong, no matter how the short circuit level is. Consequently, the whole system may not suffer any problems at all. However, such a conclusion is obviously impractical. For an ac system even with a somewhat big value of $S_{S C C}$ in the context above, a TOV problem may still be induced by adequate shunt compensation.

\subsection{Discussion about converter equivalence}

Equations of (2), (3), and (4) show the similarity in the procedure to define SCRs for corresponding HVDC system configurations. In detail, an equivalent converter is integrated firstly at the given converter station, which is performed by weighting active power of a converter with interaction factors, such as ratio of elements of node impedance matrix and MIIF. Then, the equivalent converter is dealt with like what is done with a single-infeed HVDC system, i.e. just the equivalent active power of the equivalent converter is chosen to compare with the short circuit capacity at the station.

In current researches of SCR definitions for multi-infeed HVDC systems, converters are always modeled based on the CIGRE HVDC benchmark model, and equipped with the same combination control mode of constant current control at the rectifier side and constant extinction angle control at the inverter side (CC/CEA), which makes all the inverters have similar power-voltage characteristics in both static and dynamic states. Hence, the equivalent inverter can inherit the similar power-voltage characteristics from those existing inverters through the equivalent procedure. For such a situation, the equivalent inverter is allowed to be dealt with based only on its active power, since the active power-voltage characteristic can represent the whole power-voltage characteristics of the equivalent inverter. It is verified more or less by the successful usage of (3) in multi-infeed systems.

However, for the configuration of rectifier-inverter HVDC system, an equivalent converter like that in multiinfeed HVDC system does not exist anymore, since converters in the system have different operation types and control modes, i.e. different power-voltage characteristics. Consequently, it results in a failed application of (3) in rectifier-inverter systems.

The methodology of converter equivalence has explored a new way of weighting converter active power by exponential MIIF, addressed in (4). For the way above, the value of exponent constant $\alpha$ is obtained by parameter fitting, which may put mathematically its effectivity on system parameters. Then, there is maybe inconvenience caused by revaluing $\alpha$ in new systems. Moreover, the definition of (4) is subject to optimistic system strength in the scenario where an inverter has a close electrical distance with an adjacent rectifier. Considering such problems of (4) discussed above, the paper will present a new definition for multi-converter HVDC systems based on theoretical analysis of equivalent converter, and the presentation will begin with analysis of voltage interaction between converters, due to the impact of voltage interaction on the performance of the whole system and the procedure of converter equivalence.

\section{Analysis of Voltage Interaction between Converters}

\subsection{Analysis of voltage interaction based on the analytical definition of MIIF}

An indicator known as MIIF, based on the ratio of voltage changes, is empirically defined as (5) to measure the interaction level among inverters located in a common ac system, since the performance of an inverter mostly depends on the quality of the ac voltage at its terminal [3].

$$
M I I F_{j, i}=\frac{\Delta U_{j}}{\Delta U_{i}}
$$

In (5), $\Delta U_{j}$ is the observed voltage change at the converter ac bus $j$ because of a manual minor induced voltage change $\Delta U_{i}$ at the converter ac bus $i$. Corresponding to the empirical definition of MIIF, an analytical definition of MIIF is derived from an eliminated dc-variable power flow equations [15], and given by

$$
M I I F_{j, i}=\frac{\left(\mathbf{J}_{\mathbf{R}}^{-1}\right)_{j, i}}{\left(\mathbf{J}_{\mathbf{R}}^{-1}\right)_{i, i}}
$$

where $\mathbf{J}_{\mathbf{R}}=\mathbf{J}_{\mathbf{Q V}}-\mathbf{J}_{\mathbf{Q \delta}} \mathbf{J}_{\mathbf{P \delta}}^{-1} \mathbf{J}_{\mathbf{P V}}$ is the $Q V$ Jacobian 
matrix of the power flow equations as given by (7), which represents mathematically a multi-converter HVDC system retaining only converter ac bus nodes.

$$
\left[\begin{array}{l}
\Delta \mathbf{P}_{\mathbf{t}} \\
\Delta \mathbf{Q}_{\mathbf{t}}
\end{array}\right]=\left[\begin{array}{ll}
\mathbf{J}_{\mathbf{P \delta}} & \mathbf{J}_{\mathbf{P V}} \\
\mathbf{J}_{\mathbf{Q \delta}} & \mathbf{J}_{\mathbf{Q V}}
\end{array}\right]\left[\begin{array}{l}
\Delta \delta \\
\Delta V / V
\end{array}\right]
$$

The notations in (7) are self-explanatory. In detail, $\mathbf{J}_{\mathbf{P \delta}}$ and $\mathbf{J}_{\mathbf{Q \delta}}$ have the same expressions with the sub matrixes in a similar configuration of a pure ac system, respectively, while $\mathbf{J}_{\mathbf{P V}}$ and $\mathbf{J}_{\mathbf{Q V}}$ need to add the partial derivatives of converter power with respect to its ac bus voltage, $\partial P_{d c} / \partial V_{t}$ and $\partial Q_{d c} / \partial V_{t}$, into their diagonal elements, respectively. The values of $\partial P_{d c} / \partial V_{t}$ and $\partial Q_{d c} / \partial V_{t}$ depend on the control modes employed by a $\mathrm{dc}$ link, and their expressions at common combination control modes are listed in Appendix A. Then, elements of $\mathbf{J}_{\mathbf{R}}$, as well as the value of MIIF, are related to network parameters and operation states of ac system, and powervoltage characteristics of a converter.

By the expressions of $\partial P_{d c} / \partial V_{t}$ and $\partial Q_{d c} / \partial V_{t}$ in Appendix A, it can be found that for a converter under arbitrary control modes, its reactive power-voltage characteristic, compared with its active power-voltage characteristic, has an absolutely dominant effect on MIIF. Firstly, consider situations of a rectifier with an arbitrary control mode, or of an inverter with the constant extinction angle control mode (the opposite rectifier employs the constant power control mode, i.e. CP/CEA) or the constant voltage control mode (the opposite rectifier employs the constant current control mode, i.e. $\mathrm{CC} / \mathrm{CV})$. Because $\partial P_{d c} / \partial V_{t}$ values at 0 under these control modes, MIIF is only dependent on the converter reactive power-voltage characteristic and has no relation to the converter active power-voltage characteristic.

Secondly, consider the last situation of an inverter with the constant extinction angle control mode (the opposite rectifier employs the constant power control mode, i.e. CC/CEA), wherein $\partial Q_{d c} / \partial V_{t}$ has a negative value and $\partial P_{d c} / \partial V_{t}$ has a positive value. As we know, for a pure ac high voltage network, the inter-relationships of between reactive power and bus voltage magnitude, and between active power and bus voltage angle, make the equation of (8) approximate. Then, the negative reactive powervoltage and positive active power-voltage characteristics of the inverter lead to (8) more accurate, (6) thus can be rewritten approximately as (9). Because the elements in (9) are only related to converter reactive power-voltage characteristics, MIIF is dependent on the reactive powervoltage characteristic of the inverter more than on its active power-voltage characteristic.

$$
\begin{gathered}
\mathbf{J}_{\mathbf{R}} \approx \mathbf{J}_{\mathbf{Q V}} \\
\operatorname{MIIF}_{j, i} \approx \frac{\left(\mathbf{J}_{\mathbf{Q V}}^{-1}\right)_{j, i}}{\left(\mathbf{J}_{\mathbf{Q V}}^{-1}\right)_{i, i}}
\end{gathered}
$$

Thus, the analysis result above reflects that the reactive power-voltage characteristic of a converter, compared with its active power-voltage characteristic, plays a key role in the effect of dynamics from DC controllers on the voltage interaction level.

\subsection{Comparison of two factors about voltage interaction measurement}

The factor of $\tilde{Z}_{e . i j}$ (i.e. $Z_{i j} / Z_{i i}$ ) in (2) is a static indicator which just considers the effect of network parameters of ac system on the voltage interaction level. However, the factor of MIIF is a dynamic indicator, since it additionally includes the effect of the dynamics from DC controllers on the voltage interaction level. Hence, the difference of results of voltage interaction levels obtained by the two factors with the same system configuration is dependent mostly on the converter reactive power-voltage characteristic, due to the key role of the characteristic in the effect of dynamics from DC controllers on the voltage interaction level. In this study, a detailed comparison between both factors is carried out to investigate the difference between their abilities in assessing voltage interaction level.

A typical dual-infeed HVDC system is modelled based on the CIGRE HVDC benchmark model, shown as Fig. 2 with self-explanatory notations. In Fig. 2, two HVDC links employ the same combination control mode of $\mathrm{CP} / \mathrm{CEA}$ $\left(\gamma_{0}=15^{\circ}\right)$. And the ac system is represented by a dual-port configuration. Change values of $Z_{S 1}, Z_{S 2}$, and $Z_{\text {tie }}$ to make $E S C R_{1}$ and $E S C R_{2}$ vary from 2 to 5 at an interval

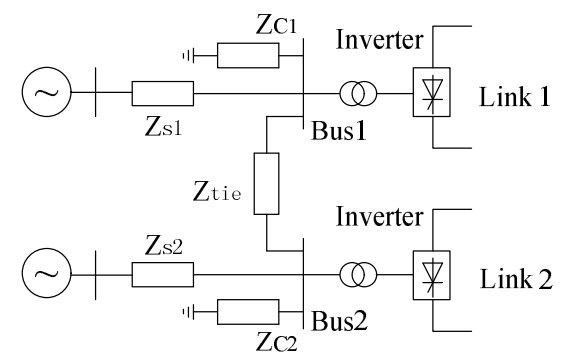

Fig. 2. The schematic diagram of a dual-infeed HVDC system

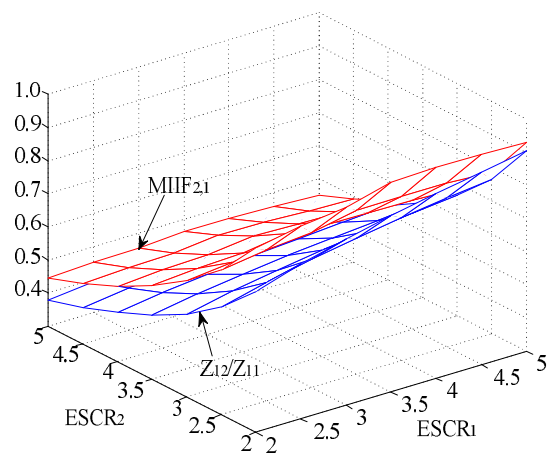

Fig. 3. Values of voltage interaction measurement factors for a dual-infeed HVDC system 
of 0.5 , accompanied always by rated voltage and rated dc power. The values of $M I I F_{2,1}$ and $Z_{12} / Z_{11}$ are simulated or calculated with the situation of disturbance brought up at the first inverter ac bus, and then plotted in Fig. 3.

Similarly, a dual-converter HVDC system with a rectifier (at Link 1) and an inverter (at Link 2) is modelled based on the CIGRE HVDC benchmark model, shown as Fig. 4. Both links employ the same control mode combination of CC/CEA $\left(\gamma_{0}=15^{\circ}\right)$. Fig. 5 plots the values of $M I I F_{1,2}$ and $Z_{21} / Z_{22}$ responding to a disturbance at the inverter ac bus.

The study results shown in Fig. 3 and Fig. 5 indicate that both of the interaction factors have the ability to reveal the general trend of voltage interaction levels along with the variation of system parameters. However, in Fig. 3, the values of $M I I F_{2,1}$ are bigger than those of $Z_{12} / Z_{11}$, due to the positive reactive power-voltage characteristic of the inverter of Link 2, which will have a more reactive consumption along with the decrease of converter ac bus voltage and then will further cause a voltage decrease. On the contrary, the values of $M I I F_{2,1}$ in Fig. 5 are smaller than those of $Z_{21} / Z_{22}$, due to a lighter power flow pressure caused by the presence of an adjacent rectifier compared with that in dual-infeed HVDC systems, and due to the negative reactive power-voltage characteristic of the rectifier of Link 1.

In [15], the mathematical derivation of (2) is carried out based on the linear network equivalence of converter power flow and with some reasonable assumptions about

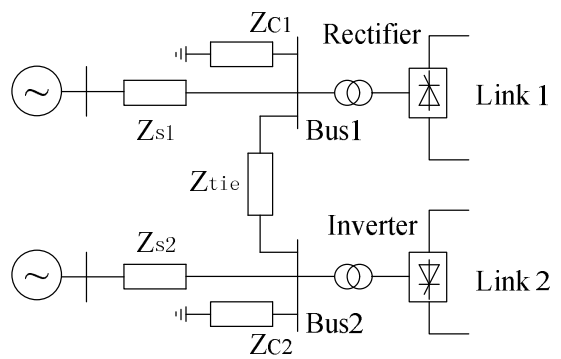

Fig. 4. The schematic diagram of a dual-convertor rectifierinverter HVDC system

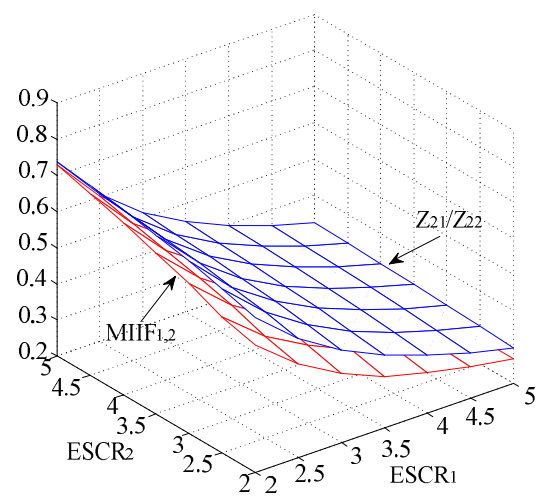

Fig. 5. Values of voltage interaction measurement factors for a dual-converter rectifier-inverter HVDC system system operating conditions, and then (2) is rewritten as (3) by ignoring dynamics from DC controllers. Such a transition of replacing $Z_{i j} / Z_{i i}$ with $M I I F_{j, i}$ in the linear equivalence of converter power flow is verified by the effectiveness in multi-infeed HVDC systems. It implies that the difference of results under the same system condition in Fig. 3 can be viewed as acceptable for qualitative studies by SCR indicators. Since the extent in the difference of results in Fig. 5 is similar to that in Fig. 3, the procedure of converter equivalence will be performed with MIIF in rectifier-inverter HVDC systems in the paper.

\section{A New Definition of SCR for Multi-converter HVDC Systems}

As discussed, the procedure of converter equivalence in a multi-infeed context may obtain an equivalent converter which can inherit similar characteristics from existing inverters. While in a rectifier-inverter context, the equivalent procedure is hard to obtain an equivalent converter like that in a multi-infeed system. The failure of (3) in rectifier-inverter systems reminds us of characterizing the equivalent converter by an additional physical quantity.

The reactive consumption of a converter has a significant effect on the system performance, especially on power transfer limit and temporary overvoltage. The amount of reactive consumption can vary greatly, depending on the converter operation type and its control mode, and the commutating reactance. Thus, an indicator which accounts for the amount of reactive consumption, named QESCR, is defined for single-infeed HVDC systems and shown as (10) [2].

$$
Q E S C R=\frac{S_{S C C}-Q_{c N}}{P_{d N}-Q_{d}}
$$

For those converters connected to a common ac system, due to the difference at operation types and control modes, they may have different static-state reactive consumption amounts, and different dynamic-state power-voltage characteristics. As analyzed in Section 3, the reactive power-voltage characteristic of a conerter, compared with its active power-voltage characteristic, has much more influence on voltage interaction between converters. It is regarded as reasonable that the physical quantity of converter reactive power should appear together with the voltage interaction factor of MIIF, especially in rectifierinverter HVDC systems, when MIIF is used to weight the impact of power flow from the converter on the strength of the common ac system.

In conclusion, the significance of the converter reactive power, discussed at both static state and dynamic state for the whole system's performance, supports strongly to take the physical quantity of converter reactive power 
into account in the definition of SCR for multi-converter HVDC systems. Then, based on the mathematical derivation of the converter equivalence in [15], a new definition is defined as (11).

$$
\operatorname{QMCESCR}_{i}=\frac{S_{S C C . i}-Q_{c . i}}{\left|\sum_{k=1}^{n} M I I F_{k, i}\left(P_{d N . k}+j Q_{d . k}\right)\right|}
$$

In Eq. (11), $Q_{d . k}$ represents the reactive consumption by the converter $k$ at rating. The directions for $P_{d N . k}$ and $Q_{d . k}$ are chosen as positive for flowing into converter bus nodes, while as negative for flowing out.

For a single-infeed HVDC system represented by the CIGRE HVDC benchmark model, in which parameters of CESCR and $Q_{d}$ value at 1.5 and 0.54 , respectively, the critical value of QMCESCR calculated by (11) is about 1.32. Thus, for a de link under consideration in a multiconverter configuration, its critical value of QMCESCR by (11) will be about 1.32 when its maximum dc power transfer occurs at dc current equal to 1.0 p.u., if the definition of QMCESCR is regarded as effective.

\section{Simulation Validation of the New Definition}

\subsection{Validation of the definition of QMCESCR for multi-infeed HVDC systems}

A dual-infeed HVDC system like Fig. 2 is modelled, in which both of two links employ the same combination control mode of $\mathrm{CC} / \mathrm{CEA}\left(\gamma_{0}=15^{\circ}\right)$. Under rated state, both inverters consume the same amount of reactive power at 0.54 p.u., while the compensation from filters and capacities at inverter side values at 0.63 p.u.

On the simulation platform of PSCAD software, dc currents of two links are increased by the same speed from 0 to about 1.10 p.u.. By choosing appropriate values of $Z_{S 1}, Z_{S 2}$, and $Z_{\text {tie }}, M A P_{1}$ occurs at $I_{d c}=1.0$ p.u. of both links. Repeat the process for several times and report values of $E S C R_{1}, E S C R_{2}, M I I F_{2,1}$ calculated by (6) and critical $Q M C E S C R_{1}$ calculated by (11) in Table 1 . In the tables below, ESCR and QMCESCR are abbreviated as ES and $\mathrm{QMC}$, respectively.

The results in Table 1 show that the critical values of QMCESCR $_{1}$ locate in the range from 1.24 to 1.38 , which has a deviation degree from $-6 \%$ to $5 \%$ around 1.32 . Theoretically, both links have the same converter powervoltage characteristics due to employing the same combination control mode, which is beneficial to obtain an equivalent converter with similar characteristics at the given converter.

Consider further the configuration of a dual-infeed link with different combination control modes, where only the inverter side of Link 2 changes to employ constant voltage
Table 1. QMCESCR values for two infeeds (Link 1 and Link 2: constant gamma)

\begin{tabular}{c|c|c|c|c|c|c}
\hline $\mathrm{Z}_{\mathrm{S} 1}$ & $\mathrm{ES}_{1}$ & $\mathrm{Z}_{\mathrm{S} 2}$ & $\mathrm{ES}_{2}$ & $\mathrm{Z}_{\text {tie }}$ & $\mathrm{MIIF}_{2,1}$ & $\mathrm{QMC}_{1}$ \\
\hline 0.47 & 2.2 & 0.53 & 2.0 & 0.86 & 0.53 & 1.26 \\
\hline 0.53 & 2.0 & 0.41 & 2.5 & 0.96 & 0.42 & 1.24 \\
\hline 0.50 & 2.5 & 0.50 & 2.5 & 0.39 & 0.69 & 1.30 \\
\hline 0.63 & 2.4 & 0.39 & 3.0 & 0.31 & 0.66 & 1.27 \\
\hline 0.36 & 3.0 & 1.09 & 2.3 & 0.14 & 1.01 & 1.31 \\
\hline 0.64 & 2.8 & 0.39 & 3.2 & 0.15 & 0.78 & 1.38 \\
\hline
\end{tabular}

Table 2. QMCESCR values for two infeeds (Link 1: constant gamma, Link 2: constant voltage)

\begin{tabular}{c|c|c|c|c|c|c}
\hline $\mathrm{Z}_{\mathrm{S} 1}$ & $\mathrm{ES}_{1}$ & $\mathrm{Z}_{\mathrm{S} 2}$ & $\mathrm{ES}_{2}$ & $\mathrm{Z}_{\text {tie }}$ & $\mathrm{MIIF}_{2,1}$ & $\mathrm{QMC}_{1}$ \\
\hline 0.47 & 2.18 & 0.53 & 1.97 & 0.93 & 0.50 & 1.28 \\
\hline 0.53 & 1.98 & 0.41 & 2.47 & 0.99 & 0.40 & 1.25 \\
\hline 0.50 & 2.46 & 0.50 & 2.46 & 0.42 & 0.62 & 1.33 \\
\hline 0.63 & 2.35 & 0.39 & 2.98 & 0.32 & 0.60 & 1.29 \\
\hline 0.36 & 2.93 & 1.09 & 2.11 & 0.19 & 0.93 & 1.33 \\
\hline 0.64 & 2.73 & 0.39 & 3.17 & 0.17 & 0.71 & 1.40 \\
\hline
\end{tabular}

control. It will cause a rated consumption amount of converter reactive power at 0.57 p.u. for the inverter of Link 2. Keep the values of $Z_{S 1}$ and $Z_{S 2}$ in Table 1 fixed, and choose appropriate values of $Z_{\text {tie }}$ to make $M A P_{1}$ occur at $I_{d c}=1.0$ p.u. for both links. Then, values of $E S C R_{1}, E S C R_{2}, M I I F_{2,1}$ and critical $Q M C E S C R_{1}$ are calculated and listed in Table 2.

According to Table 2, the critical values of $Q M C E S C R_{1}$ are around 1.32, and have a bit bigger deviation than those in Table 1. The reason is that the conversion of control modes from constant gamma to constant voltage for the inverter of Link 2 enhances the performance of dynamic voltage characteristic for the whole system. Consequently, even if with a bit weaker system strength (the same $Z_{S 1}$ and $Z_{S 2}$ while the larger $Z_{t i e}$ ), Link 1 can still reach its critical rated state with almost the same value of QMCESCR as that in Table 1.

\subsection{Validation of the definition of QMCESCR for rectifier-inverter HVDC systems}

A dual-rectifier-inverter HVDC system model like that in Fig. 3 is built. Link 1 connects into the common ac system through a rectifier while Link 2 through an inverter, and both links employ the same combination control mode of CC/CEA $\left(\gamma_{0}=15^{\circ}\right)$. The rectifier of Link 1 consumes a rated amount of reactive power at 0.53 p.u. with a rated reactive compensation amount at 0.60 p.u.. For Link 2 , the reactive power data of its inverter are the same as that in Fig. 2, i.e. 0.54 p.u. and 0.63 p.u., respectively.

The same simulation procedure is used, and appropriate values of $Z_{S 1}, Z_{S 2}$ and $Z_{\text {tie }}$ to make $M A P_{2}$ occur at $I_{d c}=1.0$ p.u. of both links are chosen. After several repeats, values of $E S C R_{1}, E S C R_{2}, M I I F_{1,2}$ and critical $Q M C E S C R_{2}$ are calculated and reported in Table 3 . The results in Table 3 show that the critical values of $Q M C E S C R_{2}$ locate in 
Table 3. QMCESCR values for dual-rectifier-inverter converters

\begin{tabular}{c|c|c|c|c|c|c}
\hline $\mathrm{Z}_{\mathrm{S} 1}$ & $\mathrm{ES}_{1}$ & $\mathrm{Z}_{\mathrm{S} 2}$ & $\mathrm{ES}_{2}$ & $\mathrm{Z}_{\text {tie }}$ & $\mathrm{MIIF}_{1,2}$ & $\mathrm{QMC}_{2}$ \\
\hline 0.75 & 1.2 & 0.75 & 1.2 & 1.43 & 0.31 & 1.21 \\
\hline 0.63 & 1.6 & 1.02 & 1.2 & 0.55 & 0.46 & 1.25 \\
\hline 0.51 & 2.0 & 0.84 & 1.4 & 0.69 & 0.34 & 1.42 \\
\hline 0.55 & 1.6 & 0.61 & 1.4 & 1.96 & 0.19 & 1.35 \\
\hline 0.68 & 1.4 & 0.66 & 1.4 & 1.24 & 0.30 & 1.41 \\
\hline 0.42 & 2.0 & 0.50 & 1.6 & 3.65 & 0.06 & 1.45 \\
\hline
\end{tabular}

Table 4. Comparison between results of (4) and (11) for dual-rectifier-inverter converters

\begin{tabular}{c|c|c|c|c|c|c}
\hline $\begin{array}{c}\mathrm{P}_{\mathrm{dN} .1} \\
(\mathrm{GW})\end{array}$ & $\mathrm{ES}_{1}$ & $\mathrm{ES}_{2}$ & $\mathrm{~L}(\mathrm{~km})$ & $\mathrm{MIIF}_{1,2}$ & $\mathrm{MI}_{2}(4)$ & $\mathrm{QMC}_{2}(11)$ \\
\hline $\mathbf{1 . 0}$ & $\mathbf{2 . 0 0}$ & $\mathbf{1 . 3 4}$ & $\mathbf{2}$ & $\mathbf{0 . 9 8}$ & $\mathbf{3 1 . 0}$ & $\mathbf{1 . 2 6}$ \\
\hline 0.2 & 6.00 & 1.15 & 2 & 0.98 & 1.42 & 1.11 \\
\hline 0.8 & 2.00 & 1.16 & 50 & 0.49 & 1.39 & 1.20 \\
\hline 1.0 & 2.00 & 1.20 & 50 & 0.42 & 1.41 & 1.25 \\
\hline 1.2 & 2.00 & 1.24 & 50 & 0.37 & 1.43 & 1.30 \\
\hline 0.6 & 1.50 & 1.15 & 80 & 0.56 & 1.38 & 1.17 \\
\hline 1.0 & 1.50 & 1.27 & 80 & 0.41 & 1.48 & 1.32 \\
\hline 0.8 & 2.00 & 1.30 & 80 & 0.40 & 1.46 & 1.32 \\
\hline 1.0 & 2.00 & 1.34 & 80 & 0.33 & 1.47 & 1.36 \\
\hline 0.8 & 2.50 & 1.35 & 80 & 0.35 & 1.47 & 1.35 \\
\hline 1.0 & 2.50 & 1.38 & 80 & 0.30 & 1.48 & 1.39 \\
\hline 0.5 & 3.00 & 1.31 & 80 & 0.46 & 1.44 & 1.29 \\
\hline 2.0 & 3.00 & 1.48 & 80 & 0.14 & 1.52 & 1.48 \\
\hline 0.5 & 3.50 & 1.35 & 80 & 0.43 & 1.46 & 1.32 \\
\hline 1.5 & 3.50 & 1.47 & 80 & 0.17 & 1.52 & 1.46 \\
\hline 0.5 & 1.50 & 1.31 & 150 & 0.46 & 1.44 & 1.29 \\
\hline 1.5 & 1.50 & 1.50 & 150 & 0.17 & 1.55 & 1.49 \\
\hline 0.5 & 2.00 & 1.37 & 150 & 0.39 & 1.46 & 1.33 \\
\hline 1.0 & 2.00 & 1.47 & 150 & 0.21 & 1.52 & 1.43 \\
\hline 3.5 & 2.00 & 1.49 & 150 & 0.06 & 1.50 & 1.45 \\
\hline 0.5 & 2.50 & 1.41 & 150 & 0.35 & 1.48 & 1.35 \\
\hline 1.5 & 2.50 & 1.50 & 150 & 0.13 & 1.53 & 1.45 \\
\hline 1.0 & 3.00 & 1.50 & 2000 & 0.01 & 1.50 & 1.32 \\
\hline & & & & & &
\end{tabular}

the range from 1.21 to 1.45 , which has a deviation degree of about $-8 \%$ to $10 \%$ from 1.32 .

A comparison between (4) and (11) is carried out as follows. The system parameters which listed in the columns from the first column to the fifth column of Table 4 , except those marked in bold italic type, are indexed directly from Table 1 in [13]. The calculation results by (4) are listed in the sixth column of Table 4. Obviously, all the results in the sixth column are the same as those in [13], except the one in bold italic type.

In the calculation of QMESCR, the amount of reactive power consumed by the rectifier of Link 1 is counted as $0.53^{*} P_{d N .1}$ MVar, and that by the inverter of Link 2 is $0.54 * P_{d N .2}$ MVar. The calculation results of QMCECSR are listed in the last column of Table 4 and plotted in Fig. 6. The values of QMCESCR in Table 4 locate in the range from 1.11 to 1.49 , and the degree of deviation from 1.32 is about from $-16 \%$ to $13 \%$. If only considering the cases with basically equivalent transportation levels, the values of QMCESCR will locate in the range from 1.20 to 1.43 , and the deviation degree is about $-9 \%$ to $8 \%$.

By Fig. 6, the deviation degrees of QMCESCR values from 1.32 are small in most cases in which the difference of power transportation levels is smaller than 0.5 p.u.. When the power difference is bigger than 0.5 p.u., the deviation degree will increase a bit. The increase of deviation degree may be caused by the reason that the converter power factor will increase for a lower power transportation level, which will result in the excess of local reactive power compensation and consequently enhances ac system strength. Then, a smaller value of QMCESCR can make the HVDC link under consideration reach earlier its MAP. While on high power transportation levels, the converter power factor will decrease and then the whole system will have a deficit of reactive power, which will cause the decline of ac system strength and consequently bring a bigger critical value of QMCESCR.

Comparisons between (4) and (11) for their applications in triple-converter systems are also made. For both tables of Table 5 and Table 6 , the system parameters in the columns of from the first column to the seventh column, and the calculation results by (4) in the eighth column are correspondingly the same as those in Table 2 and Table 3 in

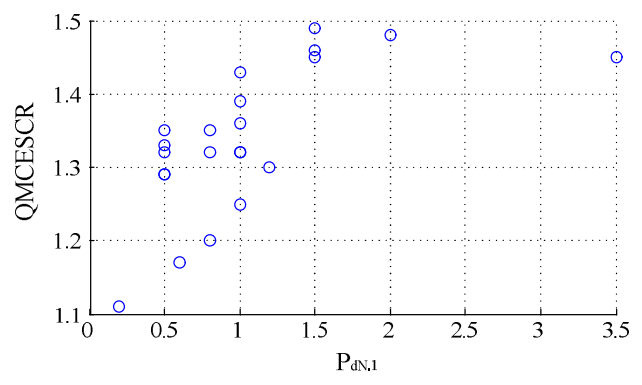

Fig. 6. The distribution diagram of the values of $\mathrm{QMCESCR}_{2}$

Table 5. Comparison between (4) and (11) for triplerectifier-inverter converters (Link 1: rectifier, Link 2 and Link 3: inverters)

\begin{tabular}{c|c|c|c|c|c|c|c|c}
\hline $\begin{array}{c}\mathrm{P}_{\mathrm{dN} .1} \\
(\mathrm{GW})\end{array}$ & $\begin{array}{c}\mathrm{P}_{\mathrm{dN} .3} \\
(\mathrm{GW})\end{array}$ & $\mathrm{ES}_{1}$ & $\mathrm{ES}_{2}$ & $\mathrm{ES}_{3}$ & $\mathrm{MIIF}_{1,2}$ & $\mathrm{MIIF}_{3,2}$ & $\begin{array}{c}\mathrm{MI}_{2} \\
(4)\end{array}$ & $\begin{array}{c}\mathrm{QMC}_{2} \\
(11)\end{array}$ \\
\hline 1.0 & 0.8 & 3.0 & 1.6 & 3.0 & 0.15 & 0.12 & 1.48 & 1.38 \\
\hline 1.2 & 0.8 & 3.0 & 1.6 & 3.0 & 0.13 & 0.11 & 1.49 & 1.39 \\
\hline 1.2 & 0.8 & 3.0 & 1.7 & 3.0 & 0.15 & 0.23 & 1.46 & 1.36 \\
\hline 1.0 & 1.2 & 2.4 & 2.2 & 1.9 & 0.57 & 0.72 & 1.40 & 1.20 \\
\hline
\end{tabular}

Table 6. Comparison between (4) and (11) for triplerectifier-inverter converters (Link 1 and Link3: rectifiers, Link 2: inverter)

\begin{tabular}{c|c|c|c|c|c|c|c|c}
\hline $\begin{array}{c}\mathrm{P}_{\mathrm{dN} .1} \\
(\mathrm{GW})\end{array}$ & $\begin{array}{c}\mathrm{P}_{\mathrm{dN} .3} \\
(\mathrm{GW})\end{array}$ & $\mathrm{ES}_{1}$ & $\mathrm{ES}_{2}$ & $\mathrm{ES}_{3}$ & $\mathrm{MIIF}_{1,2}$ & $\mathrm{MIIF}_{3,2}$ & $\begin{array}{c}\mathrm{MI}_{2} \\
(4)\end{array}$ & $\begin{array}{c}\mathrm{QMC}_{2} \\
(11)\end{array}$ \\
\hline 1.0 & 0.8 & 2.0 & 1.41 & 1.99 & 0.32 & 0.32 & 1.65 & 1.48 \\
\hline 0.8 & 0.8 & 2.3 & 1.40 & 1.98 & 0.36 & 0.32 & 1.64 & 1.47 \\
\hline 0.8 & 1.2 & 2.3 & 1.25 & 2.0 & 0.35 & 0.19 & 1.40 & 1.32 \\
\hline 0.8 & 1.2 & 2.4 & 1.1 & 2.0 & 0.49 & 0.30 & 1.47 & 1.14 \\
\hline
\end{tabular}


[13]. The results calculated by (11) are listed in the last column in Table 5 and Table 6 , respectively. Table 5 shows that the critical values of QMCESCR locate in the range from 1.20 to 1.39 , and the degree of deviation from 1.32 is about from $-9 \%$ to $5 \%$. Table 6 shows that the critical values of QMCESCR locate in the range from 1.14 to 1.48 , and the degree of deviation from 1.32 is about from $-14 \%$ to $12 \%$.

The critical values of QMCESCR in Table 1 to Table 6 for different system configurations show that the deviation degree is very small for multi-infeed systems or for rectifierinverter systems with approximate power transportation levels. While in rectifier-inverter configurations with a large difference of power transportation level, the deviation degree will increase a little bit but still be acceptable, since the SCR values are used usually to assess system performance qualitatively.

Hence, QMCESCR is a valid indicator in the evaluation of ac system strength in multi-converter HVDC systems, especially in the situations wherein an inverter is accompanied close by a rectifier. As shown in the row marked in bold italic type in Table 4, it is no doubt that the evaluation result by (11) is much more advisable than that by (4).

Moreover, it is worth emphasizing that QMCESCR, compared with the indicator of (4), can be applied conveniently in new system situations, since it is achieved based on the theoretical analysis of the converter equivalence.

\section{Simulation Validation of the New Definition in the Calculation of TOV}

TOV at the ac terminal of a converter station, due to disturbances on either the ac or dc system, is an important criterion in the design of an HVDC link. For multiconverter HVDC systems, the worst case overvoltage on the ac bus of a given converter station results from a simultaneous block of all the HVDC links in electrical proximity. A formula of TOV calculation by MIESCR in (2) is proposed for multi-infeed HVDC systems in [12], given by (12).

$$
T O V_{i}=\sqrt{1.0+\frac{2 \cdot Q_{d . i}}{M I E S C R_{i}}+\frac{1+Q_{d . i}^{2}}{M I E S C R_{i}^{2}}}-1.0
$$

Based on the similar assumptions in (12), such as purely inductive impedance of ac system and nominal power prior to simultaneous blocking for all the dc links, a similar closed-form formula to calculate TOV by QMCESCR in the context of multi-converter HVDC systems is derived, given by (13). The derivation of (13) is addressed in Appendix B.

$$
T O V_{i}=\sqrt{1+\frac{2 Q_{d c . i} / \sqrt{1+Q_{d c . i}^{2}}}{Q M C E S C R_{i}}+\frac{1}{Q M C E S C R_{i}^{2}}}-1.0
$$

In this section, the TOV induced by simultaneous blocking of all the converters is investigated for the configuration of rectifier-inverter HVDC systems, based on a dual rectifier-inverter system model similar to that in Fig. 4. Both links employ the same combination control mode of CC/CEA. The whole system operates at a rated state, in which a reactive power amount of 0.53 p.u. is consumed by the rectifier and 0.54 p.u. by the inverter. In the investigation, $E S C R_{1}$ varies from 1.5 to 5.0 at an interval of 0.5 while $E S C R_{2}$ is kept constant at 2.0. The values of TOV at Bus 1 obtained by (12) and (13) are plotted in Fig. 7 (marked as $\mathrm{TOV}_{1}$ and $\mathrm{TOV}_{2}$, respectively). As a reference, the values of TOV for a single-infeed HVDC system based on CIGRE Benchmark, which is treated as an equivalent inverter here, are also plotted in Fig. 7 (marked as $\mathrm{TOV}_{3}$ ). The closer the curve of TOV at the location of equivalent converter is to the $\mathrm{TOV}_{3}$, the better the TOV assessment is.

Analyses of the TOV values in Fig. 7 show that the formula of (13) based on QMCESCR has a better performance to assess the level of TOV than (12) based on MIESCR for rectifier-inverter HVDC systems, because the curve of $\mathrm{TOV}_{2}$ is clearly closer to the referenced curve of $\mathrm{TOV}_{3}$ than that of $\mathrm{TOV}_{1}$. For example, for a single-infeed system with ESCR at 3, TOV is at $22.2 \%$, where as in a rectifier-inverter system with $E S C R_{1}$ at 3 , the TOV on Bus 1 from blocking all converters, calculated by (13) is at $28.8 \%$ of $\mathrm{TOV}_{2}$, while calculated by (12) is at $38.5 \%$ of $\mathrm{TOV}_{1}$.

When for a multi-infeed HVDC system, (13) is still effective to assess the level of TOV, since QMCESCR will be equal to MIESCR and the latter has been proven by its successful application in that configuration.

Hence, the indicator of QMCESCR is more appropriate than the indicator of MIESCR by Eq. (3) for the assessment of TOV for multi-converter HVDC systems. Furthermore, it can be judged that the application in TOV assessment by SCR indicators exposes an additional advantage of the definition of QMCESCR over the

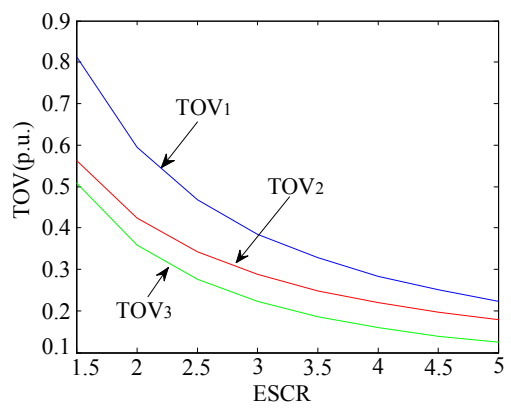

Fig. 7. Values of TOV calculated by different formulas 
definition by Eq. (4). By using formulas of (12) and (13) based on indicators of (3) and (11), respectively, the assessment of TOV can be calculated conveniently. However, for the indicator of MIESCR by Eq. (4), its application in TOV assessment probably needs a simulation, since there doesn't exist a formula like equations of (12) and (13), and thus the application is obviously not convenient.

\section{Conclusions}

In this paper, a new definition of SCR concept, which takes converter reactive consumption into account and thus named QMCESCR, is proposed for multi-converter HVDC systems. Analysis results of voltage interaction based on the analytical definition of MIIF show that the interaction level depends mostly on the reactive power-voltage characteristic of a converter compared with its active power-voltage characteristic. When the factor MIIF is used as the weighting coefficient to integrate an equivalent converter, the physical quantity of converter reactive power should be introduced into the definition of SCR concept for multi-converter systems, especially for the configuration of rectifier-inverter systems. Simulation results based on the method of MPC curve indicate that the proposed definition of QMCESCR is valid for multi-converter HVDC systems, especially in configurations wherein an inverter is accompanied close by a rectifier. Based on the proposed definition, a formula to calculate TOV is derived for multiconverter systems and then is verified.

\section{Appendix A}

The control mode at the rectifier side of a dc link could be the constant power (CP) or the constant current (CC), while the constant voltage $(\mathrm{CV})$ or the constant extinction angle (CEA) is at the inverter side. Then, possible combination control modes employed in a dc link include $\mathrm{CP} / \mathrm{CV}, \mathrm{CP} / \mathrm{CEA}$ and $\mathrm{CC} / \mathrm{CEA}$. By the stable mathematical model of a dc link shown as (A1), the expressions of $\partial P_{d c} / \partial V_{t}$ and $\partial Q_{d c} / \partial V_{t}$ are listed below.

$$
\begin{aligned}
& V_{d 0 . r}=K_{m} T_{r} V_{r} \\
& V_{d . r}=V_{d 0 . r} \cos \alpha-3 X_{r} I_{d} / \pi \\
& V_{d 0 . i}=K_{m} T_{i} V_{i} \\
& V_{d . i}=V_{d 0 . i} \cos \gamma-3 X_{i} I_{d} / \pi \\
& V_{d . r}=V_{d . i}+R I_{d} \\
& P_{d . r}=V_{d . r} I_{d} \\
& P_{d . i}=V_{d . i} I_{d} \\
& Q_{d . r}=I_{d} \times \sqrt{V_{d 0 . r}^{2}-V_{d . r}^{2}} \\
& Q_{d . i}=I_{d} \times \sqrt{V_{d 0 . i}^{2}-V_{d . i}^{2}}
\end{aligned}
$$

In (A1), $K_{m}=B \cdot 3 \sqrt{2} / \pi, \quad B$ represents the number of converter bridges, and other notations are self-explanatory.

(1) when a given converter operates as an inverter in a multi-converter HVDC system,

1) $\mathrm{CV}$ is at the inverter side, while $\mathrm{CP}$ is at the other side

$$
\begin{aligned}
& \frac{\partial P_{d . i}}{\partial V_{i}}=0 \\
& \frac{\partial Q_{d . i}}{\partial V_{i}}=-I_{d} \frac{K_{m} T_{i} V_{d 0 . i}}{\sqrt{V_{d 0 . i}^{2}-V_{d . i}^{2}}}
\end{aligned}
$$

2) $\mathrm{CEA}$ is at the inverter side, while $\mathrm{CC}$ is at the other side

$$
\begin{aligned}
& \frac{\partial P_{d . i}}{\partial V_{i}}=K_{m} T_{r} \cos \gamma_{0} \cdot I_{d} \\
& \frac{\partial Q_{d . i}}{\partial V_{i}}=-I_{d} \cdot \frac{K_{m} T_{i}\left[V_{d 0 . i}-V_{d . i} \cos \gamma_{0}\right]}{\sqrt{V_{d 0 . i}^{2}-V_{d . i}^{2}}}
\end{aligned}
$$

3) CEA is at the inverter side, while CP is at the other side

$$
\begin{aligned}
\frac{\partial P_{d . i}}{\partial V_{i}} & =-2 R I_{d} \cdot \frac{\partial I_{d}}{\partial V_{i}} \\
\frac{\partial Q_{d . i}}{\partial V_{i}} & =-I_{d} \frac{K_{m} T_{i} V_{d 0 . i}-V_{d . i} \cdot \frac{\partial V_{d . i}}{\partial V_{i}}}{\sqrt{V_{d 0 . i}^{2}-V_{d . i}^{2}}} \\
& -\sqrt{V_{d 0 . i}^{2}-V_{d . i}^{2} \frac{\partial I_{d}}{\partial V_{i}}}
\end{aligned}
$$

In (A4), $\frac{\partial I_{d}}{\partial V_{i}}=\frac{K_{m} T_{i} \cos \gamma_{0} I_{d}}{\sqrt{2} K_{m} X_{i} I_{d} / 2-2 R I_{d}-U_{d . i}}$ and

$\frac{\partial V_{d . i}}{\partial V_{i}}=K_{m} T_{i} \cos \gamma_{0}-\frac{\sqrt{2}}{2} K_{m} X_{i} \frac{\partial d I_{d}}{\partial V_{i}}$.

The expression of $\partial P_{d . i} / \partial V_{i}$ has an approximate value of 0 , since $R$ represents the resistance of DC line and has a very small value.

(2) when a given converter operates as a rectifier in a multi-converter HVDC system, for all of the three combination control modes, the expressions of $\partial P_{d c} / \partial V_{t}$ and $\partial Q_{d c} / \partial V_{t}$ are the same, respectively, shown as (A5).

$$
\begin{aligned}
& \frac{\partial P_{d . r}}{\partial V_{r}}=0 \\
& \frac{\partial Q_{d . r}}{\partial V_{r}}=-I_{d} \cdot \frac{K_{m} T_{r} V_{d 0 . r}}{\sqrt{V_{d 0 . r}^{2}-V_{d . r}^{2}}}
\end{aligned}
$$




\section{Appendix B}

Based on Fig. 1, the currents injected into a converter ac bus by the converter (named as $I_{H V D C}$ ) and by the Thevenin ac source (named as $I_{\text {Source }}$ ) are given by (B1) and (B2), respectively. $V_{t}, X_{s}, X_{f}$ and $E_{s}$ represent the converter ac bus voltage, the ac system impedance, the impedance of the filter banks and compensation capacities and the Thevenin ac source potential, respectively. In the derivation, the ac system is assumed with purely inductive impedance.

$$
\begin{gathered}
I_{H V D C}=\frac{P_{d c}+j Q_{d c}}{V_{t}} \\
I_{\text {Source }}=\frac{E_{s}}{j X_{s}}
\end{gathered}
$$

Using the KCL on the converter ac bus, (B3) can be derived.

$$
\left(\frac{1}{j X_{s}}+\frac{1}{-j X_{f}}\right) \cdot V_{t}=\overline{\left(\frac{P_{d c}+j Q_{d c}}{V_{t}}\right)}+\frac{E_{s}}{j X_{s}}
$$

Considering a multi-converter HVDC system, (B4) will be valid. In (B4), $\mathbf{Y}$ is the system admittance matrix, $\mathbf{V}_{\mathbf{t}}$ is the vector of the complex numbers for bus voltages, $\mathbf{Y}_{\mathbf{s}}$ is the vector of Thevenin inductances, and $\mathbf{E}_{\mathbf{s}}$ is the vector of Thevenin sources.

$$
\mathbf{Y} \cdot \mathbf{V}_{\mathbf{t}}=\frac{\mathbf{P}_{\mathbf{d c}}-j \mathbf{Q}_{\mathbf{d c}}}{\overline{V_{t}}}-j \mathbf{Y}_{\mathbf{s}} \cdot \mathbf{E}_{\mathbf{s}}
$$

At nominal operating conditions, $\mathbf{E}_{\mathbf{s}}$ is solved as (B5).

$$
\mathbf{E}_{\mathbf{s}}=-j \mathbf{Y}_{\mathbf{s}}^{-1}\left(\mathbf{P}_{\mathbf{d c}}-j \mathbf{Q}_{\mathbf{d c}}\right)+j \mathbf{Y}_{\mathbf{s}}^{-1} \mathbf{Y}
$$

After blocking all the dc links, $\mathbf{P}_{\mathbf{d c}}$ and $\mathbf{Q}_{\mathbf{d c}}$ become zero vectors and TOV can be calculated by the following equation:

$$
\mathbf{Y} \cdot \mathbf{V}_{\text {tov }}=-j \mathbf{Y}_{\mathbf{s}}\left[-j \mathbf{Y}_{\mathbf{s}}^{-1}\left(\mathbf{P}_{\mathrm{dc}}-j \mathbf{Q}_{\mathbf{d c}}\right)+j \mathbf{Y}_{\mathbf{s}}^{-1} \mathbf{Y}\right]
$$

The equation of (B6) can be simplified into (B7), where $\mathbf{Z}$ is the system impedance matrix, $\mathbf{Z}=\mathbf{Y}^{\mathbf{- 1}}$.

$$
\mathbf{V}_{\mathrm{tov}}=\mathbf{1}+\mathbf{Z} \cdot \mathbf{Q}_{\mathrm{dc}}+j \mathbf{Z} \cdot \mathbf{P}_{\mathrm{dc}}
$$

For the system under consideration, such as System 1, the voltage at Bus 1 after all the converters blocked is given by

$$
V_{\text {tov. } 1}=1+\sum_{k=1}^{n}\left(Z_{1 k} Q_{d c . k}\right)+j \sum_{k=1}^{n}\left(Z_{1 k} P_{d c . k}\right)
$$

Since $M I I F_{j i}$ is basically equivalent to $Z_{i j} / Z_{i i}$, (11) can be rewritten as (B9).

$$
\begin{aligned}
\operatorname{QMCESCR}_{1} & =\frac{1}{\left|\sum_{k=1}^{n} \mathrm{Z}_{k 1}\left(P_{d c . k}+j Q_{d c . k}\right)\right|} \\
& =\frac{1}{\sqrt{\left[\sum_{k=1}^{n}\left(Z_{1 k} Q_{d c . k}\right)\right]^{2}+\left[\sum_{k=1}^{n}\left(Z_{1 k} P_{d c . k}\right)\right]^{2}}}
\end{aligned}
$$

Then, (B9) can be expressed as the following

$$
\begin{aligned}
V_{\text {tov } .1} & =1+\sum_{k=1}^{n}\left(Z_{1 k} Q_{d c . k}\right)+j \sum_{k=1}^{n}\left(Z_{1 k} P_{d c . k}\right) \\
& =\sqrt{1+2 \sum_{k=1}^{n}\left(Z_{1 k} Q_{d c . k}\right)+\frac{1}{Q M C E S C R_{1}^{2}}}
\end{aligned}
$$

Assuming $Q_{d c . k} / Q_{d c .1}=P_{d c . k} / P_{d c .1}$ and taking $P_{d c .1}$ as the per-unitizing of other systems, (B11) can be derived.

$$
\sum_{k=1}^{n}\left(Z_{1 k} Q_{d c . k}\right)=Q_{d c .1} \cdot \sum_{k=1}^{n}\left(Z_{k 1} P_{d c . k}\right)
$$

Based on (B9) and (B11), (B12) is derived.

$$
\sum_{k=1}^{n}\left(Z_{1 k} Q_{d c . k}\right)=\frac{2 Q_{d .1}}{\sqrt{1+Q_{d .1}^{2}}} \cdot \frac{1}{Q M E S C R_{1}}
$$

Hence,

$$
V_{t o v .1}=\sqrt{1+\frac{2 Q_{d c .1} / \sqrt{1+Q_{d c .1}^{2}}}{Q M C E S C R_{1}}+\frac{1}{Q M C E S C R_{1}^{2}}}
$$

Similarly, for any converter bus in the multi-converter HVDC system, it can be shown that

$$
V_{\text {tov } . i}=\sqrt{1+\frac{2 Q_{d c . i} / \sqrt{1+Q_{d c . i}^{2}}}{Q M C E S C R_{i}}}+\frac{1}{Q_{M C E S C R}^{2}}
$$

Hence, the calculation formula of TOV for the converter $i$ can be addressed as the equation of (B15).

$$
T O V_{i}=\sqrt{1+\frac{2 Q_{d c . i} / \sqrt{1+Q_{d c . i}^{2}}}{Q M C E S C R_{i}}+\frac{1}{Q M C E S C R_{i}^{2}}}-1.0
$$




\section{References}

[1] W. Long, S. Nilsson, "HVDC transmission: yesterday and today", IEEE Trans. Power \& Magazine, vol. 5, pp. 22-31, Mar. 2007.

[2] "IEEE Guide for Planning DC Links Terminating at AC Locations Having Low Short-Circuit Capacities", IEEE Standard 1204, 1997, pp. 18-19.

[3] B. Davies, A. Williamson, A. M. Gole, B. Ek, B. Long, B. Burton, D. Kell, D. Brandt, D. Lee, E. Rahimi, G. Andersson, H. Chao, I. T. Fernando, K. L. Kent, K. Sobrink, M. Haeusler, N. Dhaliwal, N. Shore, P. Fischer, and S. Filizadeh, "Systems with multiple DC infeed", CIGRÉ Working Group B4.41. Dec. 2008, Publ. 364.

[4] E. Rahimi, A. M. Gole, and J. B. Davies, "Analysis of multi-infeed HVDC systems with inverter and rectifier connected to the same AC network," IET International Conference on $A C$ and DC Power Transmission, London, England, 2010.

[5] Z. Liu, Y. Biao, W. Zhang, and Y. Zhang, "Study on voltage class series for HVDC transmission system", Proceedings of the CSEE, vol. 28, pp. 1-8, Apr. 2008.

[6] D. Lee and G. Andersson, "Voltage stability analysis of multi-infeed HVDC systems," IEEE Trans. Power Del., vol. 12, no. 3, pp. 1309-1317, July. 1997.

[7] D. Lee and G. Andersson, "Power stability analysis of multi-infeed HVDC systems," IEEE Trans. Power Del., vol. 13, no. 1, pp. 923-931, July. 1998.

[8] E. Rahimi, A. M. Gole, J. B. Davies, I. T. Fernando, and K. L. Kent, "Commutation failure and overvoltage phenomena in multi-infeed HVDC systems," in Proc. Colloq. Role HVDC, FACTS Emerg. Technol. Evolving Power Syst., Bangalore, India, Sep. 2005, pp. 54-73.

[9] E. Rahimi, A. M. Gole, J. B. Davies, I. T. Fernando, and K. L. Kent, "Commutation failures analysis in multi-infeed HVDC systems," IEEE Trans. Power Del., vol. 26, no. 1, pp. 378-384, Jan. 2011.

[10] I. T. Fernando, K. L. Kent, J. B. Davis, E. Rahimi, and A. M. Gole, "Parameters for planning and evaluation of multi-infeed HVDC schemes," presented at the CIGRÉ Osaka Symp., 2007.

[11] P. F. de Toledo, B. Bergdahl, and G. Asplund, "Multiple infeed short circuit ratio - Aspects related to multiple HVDC into one AC network," in Transmission and Distribution Conference and Exhibition: Asia and Pacific, Dalian, China, Aug. 2005, pp. 1-6.

[12] E. Rahimi, "Voltage interaction and commutation failure phenomena in multi-infeed HVDC systems," Ph.D. dissertation, Dept. Elect. Comput. Eng., Univ. Manitoba, Winnipeg, MB, Canada, 2011.

[13] X. Chen, A. M. Gole, and M. Han, "Analysis of mixed inverter/rectifier multi-infeed HVDC systems," IEEE Trans. Power Del., vol. 27, no. 3, pp. 1565-
1573, Jan. 2012.

[14] M. Szechtman, T. Wess, and C. V. Thio, "First benchmark model for HVDC control studies," Proc. CIGRÉ Working Group 14.02, pp. 54-73, Apr. 1991.

[15] D. Lee and G. Andersson, "Analysis of voltage and power interactions in multi-Infeed HVDC systems," IEEE Trans. Power Del., vol. 28, no. 2, pp. 816-824, Jan. 2013.

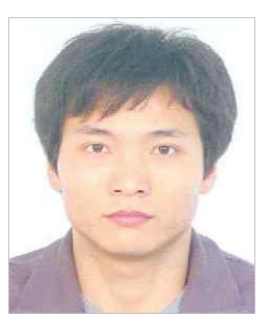

Dengfeng Liu He received the B. Eng. and the M. Eng degrees in electrical engineering from Huazhong University of Science and Technology(HUST), Wuhan, China, in 2005 and 2007, respectively. He is currently pursuing the Ph.D. degree. His research interests include fault analysis and protection setting calculation of HVDC.

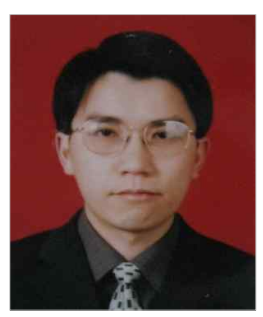

Dongyuan Shi He received the B. Eng. and the $\mathrm{Ph} . \mathrm{D}$. degrees in electrical engineering from Huazhong University of Science and Technology(HUST), Wuhan, China, in 1996 and 2002, respectively. $\mathrm{He}$ is currently an Associate Professor in the College of Electrical and Electronic Engineering at HUST. His research interests include power system analysis and application of artificial intelligence in power systems.

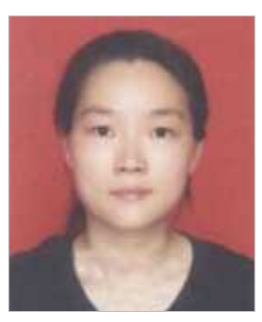

Yinhong Li She received the B. Eng. degree and the Ph.D. degree in electrical engineering from Huazhong University of Science and Technology (HUST), Wuhan, China in 1998 and 2004, respectively. Dr. $\mathrm{Li}$ is an Associate Professor in the College of Electrical and Electronic Engineering at HUST. Her research interests include relay coordination, voltage stability, and load modeling. 